DOI: $10.2478 / \mathrm{v} 10122-011-0003-8$

\title{
ON THE POSITION OF UBI WITHIN EAST CHADIC
}

\author{
VÁCLAV BLAŽEK
}

\begin{abstract}
Václav Blažek. On the Position of Ubi within East Chadic. Lingua Posnaniensis, vol. LIII (1)/2011. The Poznan Society for the Advancement of the Arts and Sciences. PL ISSN 0079-4740, ISBN 978-83-7654-140-2, pp. 41-55.
\end{abstract}

In the article the position of a recently described East Chadic language Ubi is solved. For this aim the lexicostatistic method was used. Contrary to the classification proposed in Ethnologue ${ }_{16}$, Ubi and Mawa should be classified together with Sokoro and Barain and not together with Bidiya, Dangla, Migama, etc.

Václav Blažek, Masaryk University, Brno, Czech Republic, blazek@phil.muni.cz

1. The language Ubi is spoken in Central Chad, in the prefecture Guéra, subprefecture Mongo, in southeast from the city of Tunkul, in the village Ubi and other 6 villages around it. The population of Ubi was estimated to 1.100 in 1995 (SIL). The only description of the Ubi grammar and lexicon (c. 365 words) was published by Alıo (2004). Concerning the genetic affiliation of Ubi, its East Chadic provenance is apparent. It is also unquestioned that Ubi is not in any closer relationship with Kera \& Kwang - Lele \& Kabalai - Ndam - Tumak - Sumray languages. In the most recent issue of Ethnologue (16th ed., 2009), Ubi is classified as a language closely related to Bidiya, Migama, Dangla, and especially Mawa. The model accepted in Ethnologue represents only a light modification of the model proposed by P. Newman (1977), but without Ubi. Let us compare Newman/Ethnologue model and the result of the lexicostatistical test applied to the 12 best documented East Chadic languages (BLAŽEK 2008):

Tree diagram for the East Chadic languages after Ethnologue ${ }_{16}, 2009$ (http:// www.ethnologue.com)

Scheme 1

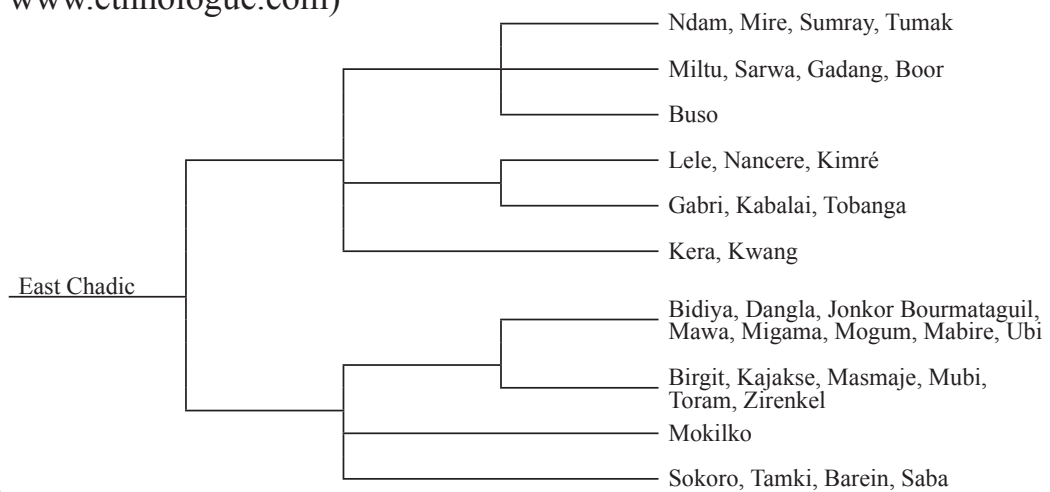


2. The percentages of common cognates for the the best documented East Chadic languages:

Table 1

\begin{tabular}{|c|c|c|c|c|c|c|c|c|c|c|c|}
\hline$[\%]$ & Lele & Sumray & Tumak & Sokoro & Ubi & Dangla & Migama & Bidiya & Mokilko & Jegu & Mubi \\
\hline Kera & 47 & 42 & 41 & 37 & 29 & 39 & 32 & 31 & 34 & 34 & 32 \\
\hline Lele & & 52 & 45 & 42 & 33 & 44 & 42 & 41 & 39 & 41 & 45 \\
\hline Sumray & & & 58 & 40 & 26 & 40 & 44 & 39 & 39 & 41 & 43 \\
\hline Tumak & & & & 40 & 33 & 42 & 35 & 35 & 36 & 41 & 38 \\
\hline Sokoro & & & & & 60 & 48 & 41 & 42 & 39 & 49 & 44 \\
\hline Ubi & & & & & & 44 & 42 & 44 & 33 & 45 & 36 \\
\hline Dangla & & & & & & & 70 & 70 & 42 & 70 & 58 \\
\hline Migama & & & & & & & & 70 & 49 & 70 & 53 \\
\hline Bidiya & & & & & & & & & 47 & 63 & 53 \\
\hline Mokilko & & & & & & & & & & 40 & 40 \\
\hline Jegu & & & & & & & & & & & 60 \\
\hline
\end{tabular}

3. Tree-diagram created for the East Chadic languages on the basis of lexicostatistics:
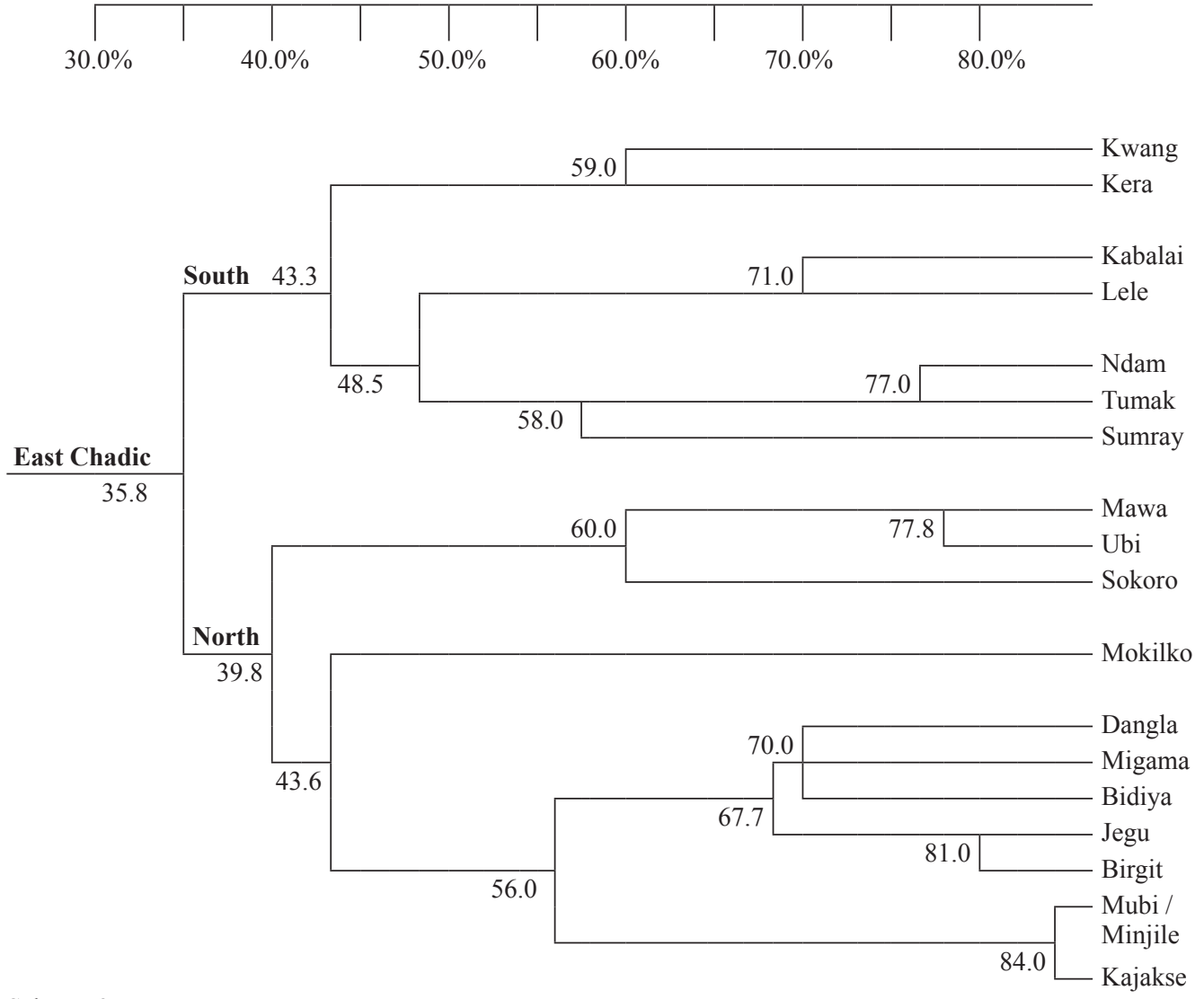

Scheme 2

Note: Toram is closer to Jegu $(65.1 \%)$ than to Mubi $(51.2 \%)$. The relatively low figures are caused by very poor Toram lexical data, $c a$. 40 items from the basic 100-word-list. 
4. Till the present time, there are only two attempts to formulate the phonetic correspondences for East Chadic languages, both in the context of the other Chadic languages: one by Jungraithmayr \& IBRISZIMOW (1994 $)$, the second by Olga Stolbova as an integral part of her Chadic Comparative Phonology (1996).

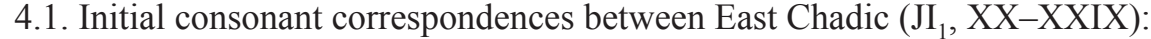

Table 2

\begin{tabular}{|c|c|c|c|c|c|c|c|c|c|c|c|c|c|c|c|c|c|c|c|c|c|c|c|c|}
\hline * & $p-$ & $6-$ & $b-$ & $b_{2^{-}}$ & $m-$ & $t-$ & $d-$ & $d-$ & $d_{2^{-}}$ & $n-$ & $k-$ & $k-$ & $g-$ & $g_{2^{-}}$ & $s_{1^{-}}$ & $s_{2}-$ & $s_{3}^{-}$ & $z-$ & $\underline{S}^{-}$ & $t_{1}-$ & $t_{2^{-}}$ & $l_{3-}$ & $l-$ & $r-$ \\
\hline $\mathrm{Kw}$ & & $b$ & $w$ & ${ }^{(m)} b$ & $m$ & $t$ & $d / d$ & & $d$ & $n$ & $k$ & $c$ & & ${ }^{n} g$ & $S$ & $s$ & $S$ & $S$ & & $S$ & $s$ & & $l$ & $t$ \\
\hline $\mathrm{Ke}$ & & 6 & $w$ & $\begin{array}{c}b / \\
6 / p\end{array}$ & $m$ & $t$ & $j$ & & & $n$ & $k$ & $h$ & $g$ & ${ }^{n} g$ & $s$ & $s$ & $S$ & $s$ & $j$ & $S$ & $s$ & & $l$ & $r$ \\
\hline $\mathrm{Kb}$ & $b$ & $b$ & $b$ & & $m$ & $d$ & $t / d$ & $t$ & ${ }^{n} d$ & $n$ & $g / k$ & '/y & $g$ & $\begin{array}{c}{ }^{(n)} g \\
/ k\end{array}$ & $s$ & $s$ & $s$ & $s$ & & $s$ & $s$ & & $l$ & $l / r$ \\
\hline Le & $b$ & $b$ & $b$ & ${ }^{(m)} b$ & $m$ & $d$ & $t / d$ & $t$ & $n / d$ & $n$ & $g$ & $' / h$ & $g / k$ & ${ }^{(n)} g$ & $s$ & $s$ & $s$ & $s$ & & $S$ & $s$ & & $l$ & $l / r$ \\
\hline $\mathrm{Su}$ & $b$ & $b$ & $b / w$ & $b$ & $m$ & $d$ & $d$ & $t$ & ${ }^{(n)} d$ & $n$ & $g$ & $g / y / j$ & $g$ & $g / k$ & $s$ & $s$ & $s$ & $s$ & $s$ & $s$ & $s / \check{s}$ & & $l$ & $r$ \\
\hline $\mathrm{Nd}$ & $b$ & & $b / w$ & $b / p$ & $m$ & $d$ & $d l^{\prime}$ & $d / j$ & & $n$ & $g / j$ & $g / j$ & $g$ & ${ }^{(n)} g$ & $s / h$ & $s$ & $h$ & $h$ & & $s / h$ & $s$ & & $l$ & $r$ \\
\hline $\mathrm{Tu}$ & & 6 & $b$ & $m$ & $m$ & $d$ & , & & & & $g$ & $g / \check{z}$ & $g$ & ${ }^{(n)} g$ & $h$ & $h$ & $h$ & $h$ & & $h$ & $h$ & & $l$ & $y / r$ \\
\hline $\mathrm{Sk}$ & $p / b$ & $f / p$ & $b$ & $\begin{array}{l}{ }^{(m)} b \\
/ m\end{array}$ & $m$ & $t$ & $d$ & $d$ & $t$ & & $g / k$ & , & $g$ & ${ }^{n} g$ & $s$ & $s$ & $s$ & $s$ & & & $s$ & & $l$ & $l / r$ \\
\hline $\mathrm{Da}$ & $p$ & $p$ & $b$ & & $m$ & $t$ & $d^{(y)}$ & $d$ & & & $k$ & $\mathrm{k} / \mathrm{g}$ & $g$ & ${ }^{(n)} g$ & $s$ & $s$ & $s$ & $z$ & & & & $s$ & $l$ & $r$ \\
\hline $\mathrm{Mg}$ & $p / b$ & $p$ & $b$ & $b$ & $m$ & $t$ & $d^{(y)}$ & $d$ & & $n$ & $k$ & $' / d^{y}$ & $g$ & $g$ & $s$ & & $s$ & $z$ & & & $c$ & & $l$ & $r$ \\
\hline $\mathrm{Mk}$ & $p$ & $b$ & $p / b$ & $m$ & $m$ & $t / d$ & & $d$ & & $n$ & $g$ & , & $g$ & $g$ & $s$ & & $s$ & $s$ & & $S$ & $s$ & & $l$ & $r$ \\
\hline $\mathrm{Jg}$ & $f$ & & $b$ & & $m$ & $t$ & $d$ & $d$ & & & $k$ &,$/ k / g$ & $g$ & ${ }^{(n)} g$ & $s$ & $s$ & $s$ & $z$ & & $s$ & $c$ & & $l$ & $r$ \\
\hline $\mathrm{Bi}$ & $f$ & & $b$ & & $m$ & $t$ & $\begin{array}{l}d^{y} \\
/,\end{array}$ & $d$ & & $n$ & $k$ & $\begin{array}{l}' / k / \\
h / j \\
\end{array}$ & $g$ & $\eta / g$ & $s$ & $s$ & $s$ & $z$ & & $s$ & $c$ & & $l$ & $r$ \\
\hline $\mathrm{Mb}$ & $f$ & & $b$ & 6 & $m$ & $t$ & $d^{(y)}$ & $d$ & & & $k$ & $k / j / \rho$ & $g$ & $\eta / k$ & $s$ & $s$ & $s$ & $j$ & & $S$ & $c$ & & $l$ & $r$ \\
\hline
\end{tabular}

Abbreviations: Bi - Birgit, Da - Dangla, Jg - Jegu, Kb - Kabalai, Ke - Kera, Kw - Kwang, Le - Lele, Mb - Mubi, Mg Migama, Mk - Mokilko, Nd - Ndam, Sk - Sokoro, Su - Sumray, Tu - Tumak

4.2. Medial and final consonant correspondences between East Chadic $\left(\mathrm{JI}_{1}, \mathrm{XX}-\right.$ XXIX):

Table 3

\begin{tabular}{|c|c|c|c|c|c|c|c|c|c|c|c|c|c|c|c|c|c|c|c|c|c|c|c|}
\hline$*$ & $-p-$ & $-6-$ & $-b-$ & $-b_{2}-$ & $-m-$ & $-t-$ & $-d-$ & $-d-$ & $-d_{2^{-}}$ & $-n-$ & $-k-$ & $-K-$ & $-g-$ & $-S_{1^{-}}$ & $-S_{2^{-}}$ & $-S_{3}-$ & $-z-$ & \begin{tabular}{|l|}
$-S-$ \\
\end{tabular} & $-t_{1^{-}}$ & $-t_{2}-$ & $-1 z^{-}$ & $-l-$ & $-r-$ \\
\hline $\mathrm{Kw}$ & $w$ & & $p$ & & $m$ & $y$ & $d$ & $d$ & $d$ & $n$ & & & & & $S$ & $s$ & & & $s$ & & & $l$ & $r$ \\
\hline $\mathrm{Ke}$ & $w$ & & & $b / m$ & $m$ & $\varnothing$ & $d / y$ & $d$ & & $n$ & $g$ & & $g$ & & $s$ & $s$ & & & $s$ & & & $l$ & $r$ \\
\hline $\mathrm{Kb}$ & $p$ & & $b$ & $p$ & $m$ & $\varnothing / d$ & $r$ & $d / r$ & ${ }^{n} d$ & $n$ & & & $g / y$ & & $s$ & $s$ & $s$ & $s$ & $s$ & $y$ & & $l$ & $r$ \\
\hline $\mathrm{Le}$ & $p$ & & $m$ & $m / b$ & $m$ & $\varnothing / d$ & $r$ & $r$ & $n / d$ & $n$ & $y$ & & $g$ & & $S$ & $S$ & & & $S$ & & & $l$ & $r$ \\
\hline $\mathrm{Su}$ & $w$ & & $b$ & & $m$ & $r / d$ & $d$ & $d$ & ${ }^{(n)} d$ & $n$ & g/Ø & $k$ & $g$ & & $S$ & $s$ & $s$ & $s$ & $s$ & & & $r / l$ & $r$ \\
\hline $\mathrm{Nd}$ & $w$ & & $b$ & $m$ & $m$ & $y / t$ & $t$ & $c / r$ & & $n$ & $g$ & & $g$ & & $h$ & $s$ & & & $h / s$ & & & $l$ & $r$ \\
\hline $\mathrm{Tu}$ & $w$ & & & & $m$ & $\varnothing$ & $r / d$ & & & $n$ & $g$ & & $g$ & & $h$ & $y$ & & & $h$ & & $l$ & $l$ & $r$ \\
\hline Sk & $p$ & & & & $m$ & $t / y$ & $\begin{array}{l}d / \\
d / t\end{array}$ & $d$ & $t$ & $n$ & & & & & $s$ & $s$ & & & & & $l$ & $l / r$ & $r$ \\
\hline $\mathrm{Da}$ & $p$ & $p$ & $p$ & & $m$ & $t$ & $d$ & $d$ & & $n$ & $k$ & $g$ & & & $s$ & $s$ & & $t^{y} / s$ & & $c$ & & $l l / r$ & $r$ \\
\hline $\mathrm{Mg}$ & $p$ & $p$ & $b / f$ & ${ }^{m} b$ & $m$ & $t$ & $d^{(v)}$ & $d$ & & $n$ & $y$ & & & & & $s / t$ & & $s$ & & $s$ & $l$ & $l / r$ & $r$ \\
\hline $\mathrm{Mk}$ & $p$ & & $b$ & & $m$ & $d / d$ & $d$ & $r / d$ & & $n$ & $\varnothing$ & $k$ & $k$ & & & $s / z$ & $z$ & $d / z$ & & & $r$ & $l$ & $r$ \\
\hline $\mathrm{Jg}$ & $f$ & & $b$ & $b$ & $m$ & $t$ & $d$ & $d$ & & $n$ & $\varnothing$ & & & & $s$ & $s$ & & $k^{v}$ & & $s$ & $l$ & $l$ & $r$ \\
\hline $\mathrm{Bi}$ & $f$ & $f$ & $b$ & & $m$ & $t$ & $d / d$ & $d$ & & $n$ & $y$ & & & & $s$ & $s$ & & $s$ & & $s$ & $l$ & $l / r$ & $r$ \\
\hline $\mathrm{Mb}$ & $f$ & 6 & $b$ & ${ }^{m} b$ & $m$ & $t / d$ & $d$ & $r$ & & $n$ & $c / y / \eta$ & & $g / \varnothing$ & & $S$ & $S$ & & $S$ & & $s$ & $l$ & $l$ & $r$ \\
\hline
\end{tabular}


4.3. Initial East Chadic consonant corresponences (without resonants) according to Stolbova (1996: 97)

Table 4

\begin{tabular}{|c|c|c|c|c|c|c|c|c|c|c|c|c|c|c|c|c|c|c|c|c|c|}
\hline $\mathrm{pECh} *$ & $p-$ & $p_{1^{-}}$ & $f-$ & $b-$ & $b-$ & $t-$ & $t-$ & $d-$ & $d-$ & $s-$ & $z-$ & $\check{c}-$ & $\check{c}_{1^{-}}$ & $\check{3}-$ & $s^{\prime}-$ & $\dot{c}-$ & $c^{\prime}-$ & $k$ - & $g-$ & $\gamma-$ & $h$ - \\
\hline pKera * & $p$ & $p_{1}$ & $f$ & $b$ & $b$ & $t$ & & $d$ & $d$ & $s$ & $z$ & $\check{c}$ & & $\check{3}$ & $l$ & \multicolumn{2}{|c|}{$s$} & $k$ & $g$ & $(\gamma)$ & $h$ \\
\hline Kera & $p^{1}$ & $f$ & $f$ & $b$ & $b$ & $t^{5}$ & & $d$ & $d$ & $s$ & $z ?$ & $\check{c}$ & & $\check{3}$ & $l$ & \multicolumn{2}{|c|}{$S$} & $k$ & $g$ & $h$ & $h,{ }^{\prime} i$ \\
\hline Kwang & $p$ & $p / b$ & $p$ & $b$ & & $t$ & & $d$ & $d$ & $s$ & $s$ & $\check{c}$ & & $\check{3}$ & $l$ & & & $k$ & $g$ & $g$ & $h,{ }^{\prime} o / w$ \\
\hline pLai * & \multicolumn{3}{|c|}{$p$} & $b$ & & \multicolumn{2}{|c|}{$t,-d-$} & $d$ & $d$ & $s$ & $s$ & \multicolumn{2}{|c|}{$\check{c}$} & $\check{3}$ & \multicolumn{3}{|c|}{$s$} & $k$ & $g$ & $h$ & $(h)$ \\
\hline Kabalai & \multicolumn{3}{|c|}{$p^{2}$} & $b$ & & \multicolumn{2}{|c|}{$t-^{6},-d$} & & & $s$ & $s$ & \multicolumn{2}{|c|}{$\check{c}$} & $\check{3}$ & \multicolumn{3}{|c|}{$s$} & $k$ & $g$ & $h$ & $h, w o$ \\
\hline Lele & \multicolumn{3}{|c|}{$p^{3}$} & $b$ & & \multicolumn{2}{|c|}{$t-,-d$} & $d$ & $d$ & $s$ & $s$ & \multicolumn{2}{|c|}{$\check{c}$} & $\check{3}$ & \multicolumn{3}{|c|}{$s$} & $k$ & $g$ & $h$ & $h, w a$ \\
\hline pSumray * & $p$ & $p_{1}$ & $p$ & $b$ & $b$ & $d$ & $t$ & $d$ & $\underline{d}$ & $s$ & $s$ & \multicolumn{2}{|c|}{$\check{c}$} & 光? & $l$ & \multicolumn{2}{|c|}{$s$} & $k$ & $g$ & $h$ & '-, (h) \\
\hline Sumray & $b^{4}$ & $p$ & & $b$ & $b$ & $d^{7}$ & $t$ & $d$ & $d$ & $s$ & $s$ & \multicolumn{2}{|c|}{$s, \check{c}$} & & $l$ & \multicolumn{2}{|c|}{$s$} & $k$ & & & '-, (h) \\
\hline Ndam & $p$ & $p$ & & $b$ & $b$ & $d$ & $t$ & $d$ & $d$ & $h$ & $h$ & \multicolumn{2}{|c|}{$\check{c}$} & & $l$ & \multicolumn{2}{|c|}{$s$} & $k$ & & $h$ & '- \\
\hline Tumak & $p$ & $p$ & & $b$ & $b$ & $d$ & $t$ & $d$ & $d$ & $h$ & $h$ & & $\check{3}$ & $l$ & \multicolumn{2}{|c|}{$h$} & $k$ & & $h$ & '-, $h$ \\
\hline Sokoro & $p$ & $?$ & $(f)$ & $b$ & $b$ & $(t)$ & $t$ & $d$ & $d, d$ & $s$ & $s$ & $\check{c}$ & $(\check{c})$ & & $l$ & \multicolumn{2}{|c|}{$s$} & $k$ & $g$ & & '- \\
\hline pDangla * & \multicolumn{3}{|c|}{$p$} & $b$ & $b$ & $t$ & & $d$ & $d$ & $s$ & $s$ & & $\check{3}$ & $l$ & $\check{c}$ & $d y$ & $k$ & \multicolumn{2}{|c|}{$g$} & '- \\
\hline Dangla & \multicolumn{3}{|c|}{$p$} & $b$ & $b$ & $t$ & & $d$ & $d$ & $s$ & $s$ & \multicolumn{2}{|c|}{ 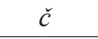 } & $\check{3}$ & $l$ & & $d y$ & $k$ & $\varepsilon$ & 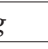 & '- \\
\hline Migama & & $p$ & & $b$ & $b$ & $t$ & & $d$ & $d$ & $s$ & $s$ & c & 5 & $\check{3}$ & $l$ & $\check{c}$ & $d y$ & $k$ & $\varepsilon$ & $g$ & '- \\
\hline Bidiya & & $p$ & & $b$ & $b$ & $t$ & & $d$ & $d$ & $s$ & $s$ & c & 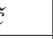 & $\check{3}$ & $l$ & $\check{c}$ & $d y$ & $k$ & $\varepsilon$ & $g$ & '- \\
\hline Mokilko & & $p$ & & $b$ & $b$ & $t$ & & $d$ & $d$ & $s$ & $s$ & $\check{c}$ & $z$ & $s$ & $l$ & $s$ & $s$ & $k$ & $\varepsilon$ & 5 & '- \\
\hline pMubi * & & $p$ & $f$ & $b$ & $b$ & $t$ & & $d$ & $d$ & $s$ & $s$ & $c$ & E & $\check{3}$ & $l$ & $\check{c}$ & $d y$ & $k$ & $\varepsilon$ & $g$ & '- \\
\hline Mubi & & $f$ & $p / f$ & $b$ & $b$ & $t$ & & $d$ & $d$ & $s$ & $s$ & $c$ & 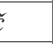 & $\check{3}$ & $l$ & & ' & $k$ & $\varepsilon$ & $g$ & $h, '-$ \\
\hline Jegu & & $p$ & $f$ & $b$ & $b$ & $t$ & & $d$ & $d$ & $s$ & $s$ & $c$ & 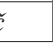 & $\check{3}$ & $l$ & & ' & $k$ & $\varepsilon$ & $g$ & '- \\
\hline
\end{tabular}

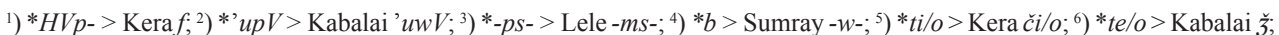
7) $* d w>$ Sumray $\left.\check{c} w ;{ }^{8}\right) * t i>$ Bidiya $\check{c} i$

4.4. Medial East Chadic consonant corresponences according to STOLBOva (1996: 144)

Table 5

\begin{tabular}{|c|c|c|c|c|c|c|c|c|c|c|c|c|c|c|c|c|c|c|c|}
\hline $\mathrm{pECh} *$ & $-p-$ & $-f-$ & $-b-$ & $-b-$ & $-t-$ & $(-t-)$ & $-d-$ & $-d-$ & $-S-$ & $-3-$ & $-\check{c}-$ & $-\check{c}_{1}^{-}$ & $-\breve{z}^{-}$ & $-S^{\prime}-$ & $-\dot{c}-$ & $-c ́$ & $-k-$ & $-k_{1}^{-}$ & $-g-$ \\
\hline pKera * & $p$ & $f$ & $b$ & $b$ & \multicolumn{2}{|c|}{$t$} & $d$ & $d$ & $s$ & $(\breve{s})$ & $\check{c}, s$ & $(\grave{c})$ & $d$ & $l$ & $s$ & & 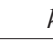 & & $g$ \\
\hline Kera & $p^{1}$ & $f$ & $b$ & $b, b$ & \multicolumn{2}{|c|}{$t$} & $d$ & $d$ & $s$ & & & $\check{c}$ & $d$ & $l$ & $s$ & & $h$ & & $g$ \\
\hline Kwang & $b$ & (b) & $b$ & & \multicolumn{2}{|c|}{$t$} & $d^{4}$ & $d$ & $s^{9}$ & & $\check{c}$ & & & $l$ & $s$ & & $h$ & & $g$ \\
\hline pLai * & \multicolumn{2}{|c|}{$? b$} & $b$ & $b$ & $d$ & $?$ & $d$ & $d$ & $s$ & $s, \check{3}$ & & $s, \check{c}$ & $s$ & & \multicolumn{2}{|c|}{$s$} & \multicolumn{2}{|c|}{$k$} & $g$ \\
\hline Kabalai & $b$ & $b^{2}$ & $b$ & & $d$ & & & $d$ & $s$ & $\check{3}$ & & & $\check{3}$ & & \multicolumn{2}{|c|}{$s$} & \multicolumn{2}{|c|}{$k$} & $g$ \\
\hline Lele & & $b$ & & $b$ & $d$ & & $d$ & $d$ & $s$ & $s$ & & & & & \multicolumn{2}{|c|}{$s$} & \multicolumn{2}{|c|}{$k$} & $g$ \\
\hline pSumray * & \multicolumn{2}{|c|}{$b$} & $b$ & $b$ & $d$ & $?$ & $d$ & $d$ & $s$ & & & (3) & (3) & $l$ & \multicolumn{2}{|c|}{$s$} & $g$ & $k$ & $g$ \\
\hline Sumray & \multicolumn{2}{|c|}{$b, w$} & & & $d^{3}$ & & $d^{5}$ & $d$ & $s$ & & & & & $l$ & \multicolumn{2}{|c|}{$s$} & $g$ & $k$ & $g$ \\
\hline Ndam & \multicolumn{2}{|c|}{$b^{2}$} & & & $d^{3}$ & & $d^{5}$ & $d$ & $s^{10}$ & & & $\check{3}$ & $\check{3}$ & $l$ & \multicolumn{2}{|c|}{$s$} & $g$ & $k$ & $g$ \\
\hline Tumak & \multicolumn{2}{|c|}{$b$} & $b$ & & $d$ & & $d^{6}$ & $d$ & $z^{11}$ & & & $\check{3}$ & $n \check{\mathfrak{Z}}$ & $l$ & \multicolumn{2}{|c|}{$s$} & $g$ & $k$ & $g$ \\
\hline Sokoro & \multicolumn{2}{|c|}{$p, f$} & $b$ & & $(t)$ & $?$ & $d$ & $d, d$ & $s$ & $\check{3}$ & & $(s)$ & $s$ & $l$ & \multicolumn{2}{|c|}{$s$} & \multicolumn{2}{|c|}{$k$} & $g$ \\
\hline Dangla & \multicolumn{2}{|c|}{$p$} & & $b$ & & & $d$ & $d$ & $s$ & $d$ & $\check{c}$ & $d y$ & & $l$ & $\check{c}$ & $d y$ & $h$ & & $g$ \\
\hline Mig. / Bid. & \multicolumn{2}{|c|}{$p$} & & $b$ & & & $d^{5}$ & $d$ & $s$ & $d$ & $\check{c}$ & $d y$ & $d$ & $l$ & $\check{c}$ & $d y$ & $h$ & & $g$ \\
\hline Mokilko & \multicolumn{2}{|c|}{$p$} & $b$ & $b, b$ & $t$ & $(d)$ & $d$ & $d$ & $z$ & $s, z$ & & & & $l$ & \multicolumn{2}{|c|}{$s$} & 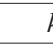 & & $g$ \\
\hline pMubi * & $p$ & $f$ & $b$ & $b$ & \multicolumn{2}{|c|}{$t$} & $d$ & $d$ & $s$ & $(d)$ & $(\check{c})$ & $d$ & & $l$ & $(\check{c})$ & $(d y)$ & $h$ & & $g$ \\
\hline Mubi & & $f$ & $b$ & $b$ & \multicolumn{2}{|c|}{ 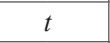 } & $d^{7}$ & $d$ & $s$ & & & $d$ & & $l$ & & '3 & $h$ & & $g$ \\
\hline Jegu & $p, f$ & $(f)$ & $b$ & & \multicolumn{2}{|c|}{$t,--\check{3}$} & $d^{8}$ & $d$ & $s$ & $d$ & $\check{c}$ & $d$ & & $l$ & & & $h$ & & $g$ \\
\hline
\end{tabular}

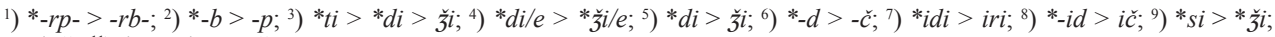
$\left.\left.{ }^{10}\right) *_{s i}>*_{-} \check{3} i ;{ }^{11}\right) *_{-s}>*_{-z}>-\check{3}$. 
4.5. The only attempt to reconstruct the Chadic correspondences in vocalism was proposed by O. Stolbova (1996: 146). She assumes the regressive vowel assimilation for the vowel of the first syllable:

Table 6

\begin{tabular}{|l|c|c|c|c|c|c|c|c|c|c|c|c|c|c|}
\hline$* \mathrm{pCh}$ & $a-o$ & $a-u$ & $a-i$ & $o-a$ & $o-u$ & $o-i$ & $u-a$ & $u-o$ & $u-i$ & $e-a$ & $e-i$ & $i-a$ & $i-o$ & $i-u$ \\
\hline Kera & $a, o$ & $a$ & $a$ & $o / a$ & $o, u$ & $o$ & $a$ & $o$ & $i$ & & & & $w a$ & \\
\hline Sumrai & $a$ & $u$ & $a / i$ & $o$ & $a, o$ & $o$ & $o / a$ & $o$ & $u$ & $a / e$ & $i$ & $i / a$ & $i / w a$ & $i, u$ \\
\hline Dangla & $o, a$ & $a$ & $a / e, i$ & $o / a, o$ & $o, a$ & $o$ & $o, a / a$ & $o$ & $i$ & $e, a$ & $e, i$ & $i, e / a$ & $o$ & $i$ \\
\hline
\end{tabular}

5. Ubi basic vocabulary with all probable or at least hypothetical cognates in other East Chadic languages

all - Ubi karaatiti, Mawa karat (R); further perhaps ?Sokoro kúldiy, Jegu kot, ?Mabire godok; Kera kede(' $i)$.

ashes - Ubi rub. Cf. WCCh: Hausa ràushii "hot ashes"; Yiwom (= Gerka) rı: $p^{h}$ "ashes" $\left(\mathrm{JI}_{2}, 4\right)$.

bark - Ubi pìrikà, Mawa purka, Sokoro fụrkía = pòrànyágá (Sx); cf. Dangla pàrdé, Migama púrdè, Bidiya pàráaḍa, ?Mokilko 'ambírsàwá. $\mathrm{JI}_{1}, 4$ derive from $\mathrm{pCh} * 6-r$ also other forms (see $\mathrm{JI}_{2}, 8-9$ ).

belly - Ubi 'atà, Mawa at (R), Barain ato, Dangla ádí = áddeń (L), Migama 'á:dí, Bidiya 'adè, Mokilko 'áddé, Mabire at, Jegu 'aad, Mubi hàt, pl. hòdút, Minjile $h_{\Lambda} d$. It is a question, if the forms from CCh quoted in $\mathrm{JI}_{2}, 20-21$ are really related: Margi $\bar{u} w \bar{u} d \bar{u}$; Bata edie (Dec); Laamang xúdì; Padukwo húdẹ, Wandala hude, Glavda xùda; Sukur xut, Mafa hwâd, Muktele hùd; Masa hạ'yạ id.

big - Ubi dèbinà.

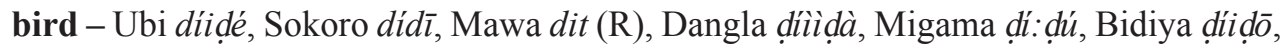

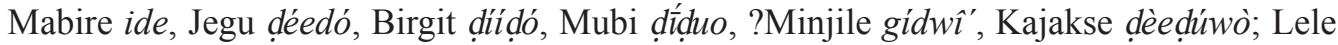
tídí, Sumray dùdí, pl. dìrbi,Tumak ḍ̀rí, maybe also Kabalai cìrró, Ndam cádíny. NEwMAN (1977: 22, \#6) reconstructs pCh *dzy-, $\mathrm{JI}_{1}, 10$ : *yd. The forms from W\&CCh see $\mathrm{JI}_{2}, 22-23$. Stolbova (1987: 240) reconstructs WCh *yadir id.

bite - Ubi 'oomin, Sokoro j̀mè (Sx), Mawa óóm, Dangla ùmè, Mabire omiə, Migama 'ó:mó, Bidiya 'om, Birgit 'ùmí ( $\mathrm{JI}_{2}, 25 ; \mathrm{JI}_{1}, 11$ : *-m).

black - Ubi cilmìn, Mawa cilim (R), Sokoro kílmio, Barain kolmo, Kujarke kálámà $u$, Mabire čilim. Stolbova (1996: 60) adds Dangla kèlmò, Migama kélèemò "shadow", reconstructing ECh *kil[e]m-; she also includes here Kera tilma "darkness", Tumak di-glàm id. etc. $\mathrm{JI}_{2}, 28-29 \& \mathrm{JI}_{1}, 13$ derive these and many other forms from $\mathrm{pCh} *_{\text {-lm }}$.

blood - Ubi seḍè, Mawa sct (R), Sokoro saā = sàw'á. The latter form resembles CCh: Kotoko Logone ski (Bouny apud $\mathrm{JI}_{2}, 31$ ). JSh 46 speculated about a metathetical cognate of WCh: Ron: Daffo \& Bokkos wash id., but in $\mathrm{JI}_{2}, 30-31$ this idea is not kept.

bone - Ubi 'èsà, Mawa ac (R), Sokoro ósseygī, Barain uosóngo, Migama 'àssú, Mokilko 'òssé, Mabire aso, Jegu 'aso, Birgit 'ásó, Kabalai ' i sí, Lele ísì, Nancere ese, Tobanga 'ạsẹ; cf. also Dangla kààsò, Bidiya káskō, Kwang kīsígī, Kera kóskón, Sumray gùsúìny, Ndam gūsé, Tumak gùūy. STolbova (1996: 123, 65-66) differentiates two pCh etymons: *'es- and *kas- (cf. also WCh *V-kasi by Stolbova 1987: 211-212), while Newman (1977: 
23, \#13) tries to derive all from a common Ch source *' Jaşu (cf. NEWMAN \& MA 1966: 241, $\# 127:{ }^{*} \underline{W-s-}$ ), while $\mathrm{JI}_{2}, 36-37 \& \mathrm{JI}_{1}, 17$ from $\mathrm{pCh} * K s_{3}$.

breast - Ubi paynà, Mabire paj. Cf. WCh: North Bauchi: Warji píyéná, Tsagu éépòn, Kariya \& Miya àpí, Pa'a ápi, Siri ípí, Mburku piìú id. (*a-pi by Stolbova 1987: 248); CCh: Zime Batna pá' $\left(\mathrm{JI}_{2}, 46-47 ; \mathrm{JI}_{1}, 20\right.$ connect it with other forms from all Chadic branches and derive all from $\mathrm{pCh} * p-6$.

burn - Ubi 'òogin, Mawa əəgəך (R), Sokoro ígide, Migama 'ìggò, Mabire iga, Mokilko 'óggè; ?Kera kí; cf. CCh: Zime Batna yé', Musgu y`id. $\left(\mathrm{JI}_{2}, 55 ; \mathrm{JI}_{1}, 24\right.$ : *yg, but without Sokoro \& Kera).

\section{claw - \\ cloud -}

cold - Ubi keckeci, Minjile ks:súk; cf. Mubi kùsúk "cold wind" and WCh: Gwandara àkúshúka "cold" ( $\mathrm{JI}_{2}, 78,81 ; \mathrm{JI}_{1}, 37$ add Sokoro ósso; Jegu 'és “cold”).

come - Ubi 'èebin, Mawa ooboy (R), Sokoro óbē; Kera bì. Cf. WCh: Tal baa ; Kulere bo; Warji buw, Tsagu bàà-, Kariya bū, Miya bu-, Mburku bùù-, Jimbin bòò- (StolbovA 1987: 248: North Bauchi *ba); CCh: ?Tera 6a; Zime Batna mbù, Kulung ba id. $\left(\mathrm{JI}_{1}, 38 ; \mathrm{JI}_{2}\right.$, 82-83; Newman \& Ma 1966: 235, \#42 include also WCh: Kanakuru 6ə; ECh: Mubi 6a, 6ów "to go"; JI 2,162 quote WCh: Tsagu b̀̀-, Kariya, Miya, Mburku, Jimbin ba-; CCh: Musgoy, Kola $v a$, Daba va, vi; Muzgu v'; ECh: Sokoro bā, be $(\mathrm{L})=6 \check{e} y(\mathrm{Sx})$ "to go".

\section{die -}

dog - Ubi kuyù, Mawa kuy (R), Sokoro kúyo; Kwang kìye, Kera kóoyá; further perhaps Dangla kànyà, Migama kânnyà, Mabire kan̆, Jegu kány, Birgit kájày, or Mokilko gédè; Nancere gerá, Gabri gia, Kabalai gàrà, Lele gìrà, Sumray dògádì, pl. gárádē, Ndam gáy, Tumak gá, pl. garák. NewmAn (1977: 25, \#37) reconstructs pCh *kar- “dog”, compatible with WCh $* k^{w / y}$ arA (Stolbova 1987: 210). $\mathrm{JI}_{1}, 49 \& \mathrm{JI}_{2}, 106-107$ try to derive almost all forms from $\mathrm{pCh} * k d n$, but they admit that there were more different root of the type $*_{-} d$, *kany or *yn. They also mention the Saharan parallels: Kanuri keri, Tubu kedi.

drink - Ubi sayin, Sokoro sa, Mawa sê = seay (R), Dangla sćc், Jegu s-, Birgit sáyà, Toram he, Mubi súwà, Minjile sūk, Kajakse suy, Kwang sèē, Kera sé, Nancere suə, Gabri so, Kabalai sùwa, Lele sì, Sumray shì, Ndam sāgâ, Tumak hè < pCh *sa/i (Stolbova 1996: 58; Ead. 1987, 180: WCh *sa/ih) $=*_{s a}$ (NEwMAn 1977: 25, \#39; NewmAn \& MA 1966: 234, $\# 23)=*_{s_{2}} w$ - $\left(\mathrm{JI}_{1}, 51\right)$. Mokilko síbè "to drink" more probably belongs to the root *sa6ə "to suck" (NEWMAn 1977: 32, \#125; $\mathrm{JI}_{1}, 160: *_{s_{2}} b_{(2)}$; otherwise Stolbova 1996: 102, reconstructing $\mathrm{pCh} * s o b-)$.

dry - Ubi 'ìyo, Mawa iyo, cf. iì “to dry”, Sokoro ie, îiy, maybe Tobanga hāy-, Gabri \& Kabalai v. haiua, Nancere v. haio, Dormo v. hai, Mokilko kûyìrày; Kwang komiékin, Kera keeje.

ear - Ubi 'oniyà, Sokoro on, besides Mokilko kùude, pl. kùwwá < *kuum-, Kajakse kôymi, pl. kuyòm (A), Kujarke kumayo; perhaps from *kV-sum-, attested without the $k$-prefix in Mubi súmá̀mù, Minjile sumaamo; Kwang sèmdî, Kera kósón, Nancere semáng, Gabri somain, Dormo sumánu, Kabalai sàmí, Lele sùmá, Sumray súmí, Ndam hăm, Tumak him, Sarwa simedam < pCh *sim-/*ku-sim- (Stolbova 1996: 56-57; Ead. 209: WCh *k/küma). Newman (1977: 25, \#40) reconstructs pCh *şəmi. $\mathrm{JI}_{1}, 53$ offer two variants $* \mathrm{~km} \& * \mathrm{~lm}$. 


\section{earth -}

eat - Ubi tîn, Sokoro táágo, Mawa tê = teay (R), Dangla tèè, Migama tíyáw, Bidiya taa, Mabire taka, Jegu $t$-, Birgit táyà, Toram ta, Mubi túwà, Minjile tūk, Kajakse $t u$, Kujarke tuye < pCh *ti (Newman 1977: 25, \#41; Newman \& Ma 1966: 234, \#25) =*twy $\left(\mathrm{JI}_{1}, 56 ; \mathrm{JI}_{2}\right.$, $120-121)$.

egg - Ubi ḍeezè, Sokoro désē = ḍèc่sć(L), Mawa deas (R), Dangla dịìsà, Migama ḍế:sè,

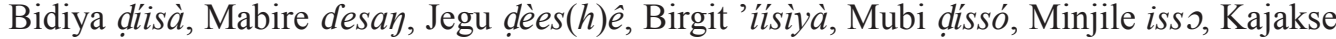
isò; Kwang tásāynká \& sē:gá, Kera kacókí, Kabalai kàs ̀̀rəy, Gabri kasire, Lele kàsìrà, Sumray gàsâ, Ndam nā:s = gae (GD), Tumak yàá, Sarwa nanas. Newman (1977: 25, \#42) reconstructs $\mathrm{pCh} *$ aşi, but the ECh forms are omitted. This protoform is compatible with WCh $*_{s a h} i$ by Stolbova 1987: $180 . \mathrm{JI}_{2}, 122-123 \& \mathrm{JI}_{1}, 57$ try to derive almost all forms from $\mathrm{pCh} * d r t$.

eye - Ubi 'irì, Sokoro íd-im = irí (B), Mawa 'ir : 'ídim "thy eye", Dangla ódò, Migama 'ídè, Bidiya 'ùdíyà, Mokilko 'êrsá, pl. 'édá, Mabire ida', Jegu 'údê, Birgit 'údì, Toram 'ùdò, Mubi írín, pl. áràn, Minjile irinî, Kajakse áríin; Kwang tè: đî̀ \& téení = k-éd-um (L), Kera ḋ̀r, Nancere téreng, Gabri tindin (L) = kindi (GD), Tobanga tiï, Dormo tine, Kabalai cidi = cindí (L), Sumray dùdí, Ndam cî, Tumak tùwór < *tu-’ad, Sarwa dèm. Stolbova (1996: 108-109): pCh *'idan- (such forms as Buduma yél, Musgu aráy, Lame irí, Masa íra-no, Zime Batna 'ir are excluded as the cognates of Egyptian ir.t id.; cf. also WCh *'ida by StoLBova 1987: 230) = *ido (Newman 1977: 26, \#46; Newman \& MA 1966: 234, \#29) = *ydn $\left(\mathrm{JI}_{2}, 126-127 ; \mathrm{JI}_{1}, 60\right)$.

fat - Ubi suunè “oil”, Sokoro súnē adj., Mawa suun "oil”, Migama séwén ká tàltà, lit. "hard oil", súúná adj., Bidiya suun "to fat", sewèy "oil”, Mabire sewę id., Mubi síwín "fat", Minjile sîwín, Kajakse síbìn = sawni (A), ?Kujarke kuno; Kwang súwānē kònà keèsū, Kera son, Kabalai sùwòngó, Lele sì̀ sòngèy sòngò (WP), Tumak ho: : $n<\mathrm{ECh} *$ siwan- < pCh *siman- (Stolbova 1996: 135) = ECh *swn "fat" = pCh *swn "oil" ( JI $_{1}, 63,130 ; \mathrm{JI}_{2}, 133$, 260-261: “oil”").

fire - Ubi 'ákò, Sokoro óko, Mawa ak, Barain aka, Dangla ako, Migama ókkò, Bidiya 'ókkò, Mabire $o k o$, Jegu 'jok, Birgit 'àkù, Toram 'ok, and maybe further Mokilko 'ùwwó, Mubi $k \dot{\varepsilon} w \bar{l}$, Minjile $k \Lambda w \hat{l}$, Kajakse $k \Lambda w i$, ?Kujarke tawe. Stolbova (1996: 81 and 32) differentiates pCh *'akuw- and *' $u w$-. Newman (1977: 26, \#48) reconstructs only pCh *aku/*akw $=*_{-} k w\left(\mathrm{JI}_{1}, 66 ; \mathrm{JI}_{2}, 138-139\right.$; Mubi \& Mokilko are included too).

fish - Ubi búuze, Sokoro bús̄̄, Mawa bùs, Barain búsi, Dangla bōosō (Lw), Migama bû:sú, Bidiya bóosà, Mabire bis, Jegu bíshó, Birgit bìsó, Mubi bògòsò, Minjile bo:gòsò, Kajakse búngòsí, Kujarke bùjá; ?Ndam bá. $\mathrm{JI}_{2}, 141$ \& $\mathrm{JI}_{1}, 67$ reconstruct $\mathrm{pCh}$ *bgs, including such forms as WCh: Daffo \& Bokkos gùshé. On the other hand, they separate WCh: SBauchi: Wangday Gàs id., connecting it with Tala kwási, Geji kwesi, Tule gìwshi, Zaar gya:s etc.

\section{five -}

fly (n.) - Ubi dòwa, Sokoro dóóu, Mawa dəw (R), Dangla díwò, Migama dìwwú, Bidiya 'úduwo, Mokilko túúdè, Jegu diwo, Birgit díwó, Mubi ḍùwó, Minjile duwòs, Kajakse tiwíyò, ?Kujarke kibiya; Sumray dôny, Ndam dúgé, Tumak dugón < pCh *diwa (Newman 1977: 26, \#52). Regarding the forms with the initial $K$ - as Hausa Kúdáá/Kújèè, $\mathrm{JI}_{1}, 71$ \& $\mathrm{JI}_{2}, 148-149$ reconstruct $\mathrm{pCh} * K d b$. 
foot - Ubi déenà, Mawa dam (R), ?Barain njidanga; cf. Kwang déenā "to come"?

four - Ubi poḍa, Mawa pat, Sokoro pááda (N), Barain fudu, Dangla pòọ̀, Migama pó:ḍi, Bidiya paaḍay, Mokilko pị̣é, Mogum pot, Mabire pot, Jegu food, Birgit fòọ̀i, Toram fòoḍa, Mubi fáḍà, Kajakse faat (A); Kwang wùḍ̂y 'ùḍ̀y, Kera wa:ḍe, Nancere pori,

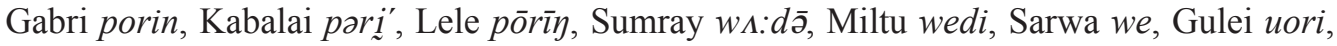
Ndam wè:tī, Tumak wari. Stolbova (1996: 152) reconstructs pCh *fid-oḍ- (but Stolbova 1987: 160 reconstructed WCh *firadu); Newman (1977: 26, \#54): *fwadə; $\mathrm{JI}_{1}, 73$ \& $\mathrm{JI}_{2}$, 152-153: *-pd.

full - Ubi munnà, Mawa muno (R), Sokoro ménna, Dangla v. mìnè, Mabire minan, Jegu mínân, Mubi mínân < ECh *mn (JSh 115).

give - Ubi birìn, ?Sokoro ̀̀rè, Dangla bérè, Migama bíráw, Bidiya bèr, Mabire birka, Jegu bir, Birgit bìrì, Mubi báárá, Kajakse faaro, Kujarke buruno; Kwang wúr, Tobanga bó, Kabalai bí, Lele bè, Sumray wúr, ?Ndam wúddíi. Stolbova (1996: 25-26) reconstructs pCh *-bari-/*-biri- (Ead. 1987, 154: WCh *ba(-)r) = *barə (NEWMAN 1977: 27, \#57; NewMAN \& MA 1966: 235, \#41) =*br $\left(\mathrm{JI}_{1}, 76 \& \mathrm{JI}_{2}, 158-58\right)$.

good - Ubi 'ulnyà; cf. Tumak ele? JSh 122 reconstructed CCh *lw "good" on the basis of Musgoy lặw "good” and Gidar leẅi "well” (see Mouchet 1950: 55).

grass - Ubi 'òozò, Mawa os (R), Sokoro ússi, Bidiya 'àwso. JSh 123 find cognates in WCh: NBauchi: Warji wasə-na, Mburku wāsə, Miya awasu, Jimbin awaši, Kariya wasisi, Diri asa' atu "spear grass" (SKInNer 1977: 24; Stolbova 1987: 260: *awasi) and CCh: Masa usna, Banana ùs inà "grass" (Kraft).

hair - Ubi lòwà, Mawa lawa (R), Sokoro lúer = làwòr (Sx), Barain lấwi, Dangla láwà, Bidiya laláawà, Mokilko 'ilill-só, pl. 'ilàlí, Jegu láawó, Toram laawa, Mubi álè, Minjile 'ale, Kajakse lày $(\mathrm{A})<\mathrm{ECh} * \operatorname{lw} r\left(\mathrm{JI}_{1}, 85 \& \mathrm{JI}_{2}, 177\right)$.

hand - Ubi kòyà, Mawa kwayidam (R) - related to Birgit jùgó, Mubi kè̀g $\frac{1}{\bar{l}}$, maybe Tumak gēn, or to Kwang kósī, Kera kasi? JSh 129 derived Kwang \& Kera forms from *k-sr, but $\mathrm{JI}_{1}, 87$ propose another solution: *kws $<* k-p s$.

head - Ubi goonà, Sokoro góndu = gon $(\mathrm{Sx})$, Mawa gwám = gwan $(\mathrm{R})$, maybe Barain kīgo, besides Dangla káá = kānn (L), Migama kàyà, Bidiya kaa-te, Mokilko 'éègó, pl. 'âw'i (LuKAs 1977: 13, 21 proposes the root 'êe-, the nominal suffix -go and the plural marker -w-; the root has to correspond to Hausa kâi "head"); Mabire kajat, Jegu kàatà, pl. kíyàu, Birgit kádyà, Toram kàata, Mubi kàc, pl. kò'ic, Minjile kac, Kajakse kàc, pl. kaụ̂úk (A), Kujarke ákè; Kera cə, Gabri cain (L), Tobanga cà, pl. caňən, Kabalai cà = cai (L), Lele cà; and with

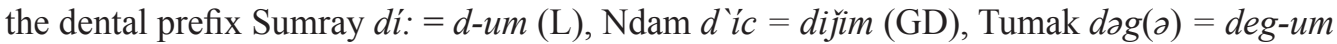
(GD), Sarwa ndim. Stolbova (1996: 31) differentiates ECh *'eg- \& *tV-eg- "head" and ECh *gon- \& *tV-gon- "occiput", although admits their relationship. Newman (1977: 27, \#67) proposes that pCh *ka was most archaic, f. also WCh *ka[y]A (Stolbova 1987: 211); in some groups of the $\mathrm{CCh}$ it was replaced by the forms derivable from $* g-n . \mathrm{JI}_{1}, 89 \& \mathrm{JI}_{2}$, 182-183 try to find a common denominator in $\mathrm{pCh} * k d n$. Regarding such forms as Mawa gwam, it is tempting to seek cognates in WCh: North Bauchi: Warji yamai, Miya, Kariya yam, Diri ama, Pa'a ḩama, Mburku gamo, Tsagu àmai, Siri yami, Jimbin gama (Skinner); South Bauchi: Seyanchi gàm, Dwot gəm, Polchi gavam, Burma kàm id. (Kraft), for which Stolbova 1987: 226 reconstructed WCh *hama. 
hear - Ubi nìyin, Mawa nïy (R), Sokoro né́yo, ?Migama gîyyò.

heart - Ubi 'oyilà, Dangla kōrlò (Lw), Migama kórlá, Bidiya kogila, Mubi kj̀rlj, Minjile ksrlò, Kajakse kırlo, Kujarke karla; Lele mú-gúlú; outside ECh perhaps CCh: Gidar ma kro "heart" : mókro "breast, chest" (Mouchet; JSh 134: *(m-)kd).

horn - Ubi gàpà, Mawa gap (R), Sokoro gẹbel = gàpón (Sx), Dangla gèćpi, Migama gâ:pè, Bidiya geepínò, Mokilko 'òpi-só, pl. 'òmbá, Mabire gepo, Jegu gééfó, Birgit géefó, Mubi gébí, pl. gà́bàb, Minjile gēbí, Kujarke gaffa; Kera gàw, Lele kēbēndí; cf. CCh: Gudu njep id. < pCh *gbn $\left(\mathrm{JI}_{1}, 94\right.$ \& $\mathrm{JI}_{2}, 192-193:$ plus Hausa Kàhóó \& Kàfóó, but this form together with South Bauchi cognates as Seyanchi kàpu, kafo etc., reflex WCh *kafu; see Stolbova 1987: 211).

I - Ubi naa, Sokoro na, Mawa nó, Dangla noon(o), Migama náà, Bidiya noo, Mokilko nùunó, Mabire na, Jegu nôo, Mubi nde, Minjile ndé, Kajakse inti, obj. 'in, Kujarke annu; Kwang 'ń, Kera ten, Tobanga $\underline{n} n \bar{u}$, Lele dàng, subj. nè (WP), Sumray j̀ndî = indī (L), Tumak subj. nò.

kill - Ubi dèyin, Sokoro da, Mawa dê = deaך (R), Dangla déć, Migama díyáw, Bidiya daa, Mokilko tóww-, pf. -ì̀d (L), Jegu $d$-, Birgit dááyà, Mubi dī, Minjile dúuk, Kajakse diwa, Kujarke duda; Sumray 'yó, Ndam 'ójâ, Tumak áj<pCh *d-(Newman 1977: 28, \#75). $\mathrm{JI}_{1}, 105 \& \mathrm{JI}_{2}, 212-213$ reconstruct $\mathrm{pCh} * d w k$, but the Sumray, Ndam, \& Tumak forms are separated.

knee - Ubi dèrnílà, Sokoro dérgel-d-ụm, Mawa dírgíl = dargal (R). Maybe related to CCh: Bata dogé, Bacama dùgé $\left(\mathrm{JI}_{2}, 215\right)$.

know - Ubi 'ùdùmin, Mawa idìm = idimin (R). Could it be related to the lexeme "eye"? Mubi yā, Minjile yeat, Kajakse yoomdi "to know" stand apparently far.

leaf - Ubi kabila. If $k a$ - is (the masculine?) prefix, it could be related to Mubi béríyò, ?Minjile berrîo, ?Kajakse fiyayò; Sumray bàje.

lie (down) -

liver - Ubi tòoziyò, Mawa tisa (R); cf. WCh: NBauchi: Tsagu ti's ə̄rəse "kidney", per-

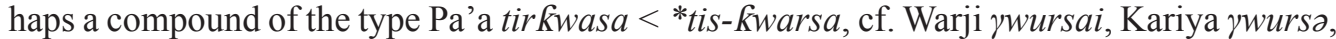
and further Ngizim kursaasiya id. (SkInNER 1977: 28; STolbova 1987: 166: WCh *a-tirsa). JSh 167: WCh *ts.

long - Ubi jaggà, Mawa jeadi (R).

\section{louse -}

man - Ubi mòotá, Mawa mota (R), Sokoro mátī, Dangla mítikò, Migama mì:dì, Bidiya miidò, Mabire mit, Jegu mitó, Birgit mìiwó, Toram miito, meetit < pCh *matu "person" (Newman 1977: 30, \#96) = *mtm $\left(\mathrm{JI}_{1}, 114-115,134\right.$ \& $\left.\mathrm{JI}_{2}, 230-231,266-267\right)$.

many - Ubi reeti, Mawa rin $(\mathrm{R})$, ?Jegu rén.

meat - Ubi biigi, Sokoro biki, Mawa biik = bik(J)< ECh *bk (JSh 177).

moon - Ubi pidimòtà.

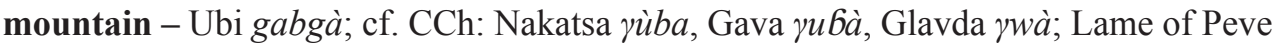
gwoi", Misme of Zime goy "mountain, stone" (Kraft).

mouth - Ubi bùu-rùm, pl. bùu-reetì, Sokoro bót-ụm, Mawa bút, Barain búja, further Dangla bïi, pl. binákí (Lw), Bidiya biite, Mokilko bizé, Mabire beto, Jegu bèetó, pl. béyàu, Birgit $b i$, Toram $b e$, Mubi bày, Kajakse $f \wedge \eta$; ?Lele kùb, Sumray $b i=b$-um (L), Ndam $b \bar{g} g=$ 
b-um (GD), Tumak bòg = bug-um (GD) < pCh *ba (Newman 1977: 29, \#88) $=* b k\left(\mathrm{JI}_{1}, 122\right.$ \& $\left.\mathrm{JI}_{2}, 244-245\right)$, cf. WCh *ba-ki (Stolbova 1987: 152).

name - Ubi sèemò, Sokoro óssō, indiv. sìntà $(\mathrm{Sx})$, Mawa sùùn = sun $(\mathrm{R})$, Dangla sîy, Migama pl. sémè, Bidiya seme, Mokilko sùmá, Birgit 'ósòm, Mubi sàmì, Minjile semmî, Kajakse simi; Kwang kás ōm sěm, Kera sám, Kabalai kosí, ?Lele kōndī, Sumray súmí, Ndam

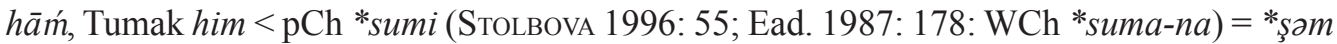
(Newman 1977: 29, \#90; Newman \& Ma 1966: 237, \#70) $=*_{s_{3}} m\left(\mathrm{JI}_{1}, 124 \& \mathrm{JI}_{2}, 248-249\right)$.

neck - Ubi jereyà, Sokoro gèr $\dot{\varepsilon}(\mathrm{Sx})=$ gét-im, Mawa jérâ : jédî 'his neck' = jed $(\mathrm{R})$, Mabire dżrea "throat”, Dangla gááḍyá = gáájeń (L) "neck”, Bidiya gè'; Tumak geér; perhaps also Mokilko góllá id. reflect pCh *giHad-id. (Stolbova 1996: 109), while Mokilko 'óré, Migama úrè, Mabire wered, Jegu were, Birgit 'úrèy, Toram were, Mubi wì̄r, Minjile wî̀i, Kajakse wuriik; Kwang kə̀rí, Kera kur, Dormo-Tobanga kornu, Kabalai kùwòsi, Lele kòrgà, Ndam kwàd = kur-um (GD) represent the derivatives of pCh *(ha-/ka-)wuyar "neck"

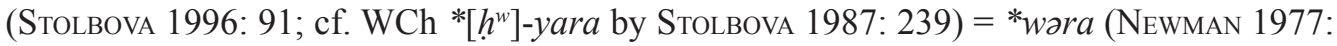
29, \#91; NewMAn \& MA 1966: 237, \#71). JI 126 \& JI 2 , 252-253 derive almost all Ch forms from $\mathrm{pCh} g d^{y} r$. The examples from Mabire and perhaps Mokilko indicate that two different lexems are here.

new - Ubi 'èrwa, Mawa oru (R); further perhaps Mubi rèwét (J), Minjile -rewèt, and Mokilko 'áwàré, if it does not reflect *'amar-; Kabalai hùrrùwă, Lele hìrwà; the forms as Dangla màrbìntò, Birgit màrìyùntà; Kwang múrwē, Kera ki-mirwí/te-merwá, are derived from pCh *m[a]ru-hu (Stolbova 1996: 133; cf. WCh *marA by Stolbova 1987: 233). Ndam $k \bar{m}$, Tumak kàmáòn may belong here too. $\mathrm{JI}_{1}, 127$ \& $\mathrm{JI}_{2}, 254-255$ all connect under the protoform *mrb.

night - Ubi bògùm, ?Sokoro bádem = bàḍúm (Sx), Mawa badəy (R). Stolbova (1996: 22) reconstructs pCh *-bur-oḍi (Ead. 1987: 154: WCh *bardi) = *badi (Newman 1977: 29, \#92), both without any ECh examples (NEwman \& MA 1966: 237, \#72 included Mubi dedem). $\mathrm{JI}_{1}, 128 \& \mathrm{JI}_{2}, 256-257$ include Sokoro, deriving all from $\mathrm{pCh} * b d$.

nose - Ubi dumilà, Sokoro dimmol, Mawa demwâl = demel (R), Mabire dəməl. $\mathrm{JI}_{2}, 259$ $\& \mathrm{JI}_{1}, 129$ derive the Sokoro form from $\mathrm{pCh} *_{n t n}$ without any comment.

one - Ubi pindarì, Barain fani, Mawa pwònnì = pənni (R), Barain fani, further Mubi finét, finí, Minjile fìnì, Kajakse finè; Nancere pená, Gabri penda (Dec) = pena $\sim$ puna (Bruel), Tobanga pòná, Dormo penā, Kabalai pònà, Lele pìnà < pCh *pn ( $\mathrm{JI}_{1}, 131$ \& JI $\left.\mathrm{J}_{2}, 262-263\right)$; JSh 198 indicate the cognates in WCh: Ron: Kulere of Ambul fáámi "1" (vs. 'ámi in other dialects) and CCh: Chibak patù, Higi Ghye paðє, reconstructing the skeleton *pn $(t)$.

person - Ubi bò, Sokoro bō, Mawa bò; Lele bāyndí, Ndam bìr id., and Gabri (L) barua, Dormo bara "man". $\mathrm{JI}_{1}, 134-135 \& \mathrm{JI}_{2}, 266-267$ quote some parallels from other branches: WCh: North Bauchi: Mburku bârgí; South Bauchi: Tala mbar, Geji mbalîn, Kir mbə̀ram; CCh: Bacama 6wáàrá; Gisiga mburo; Kotoko Logone (L) bə̀là id., all from pCh ${ }^{*} b_{2} l n$, while JSh 202 reconstructed ${ }^{* m} b r$. It agrees better with the reconstruction of STOLBOVA (1987, 154): WCh *bari "man, person".

rain $_{1}$ - Ubi pùrgum.

$\operatorname{rain}_{2}$ - Ubi 'àmyò (also “water”), Mawa ámî (also “water”); further Bidiya 'àmay (also 
“water”); Kwang kə̀miny, Nancere kúma, Dormo kumu, Kabalai kumugo (L), Lele kūmnō (WP), Tumak ná:m- (see also "water").

red - Ubi ràabìn, Mawa raabi (R), perhaps Sokoro ráyo, Mubi rènéét, or via metathesis Bidiya barga : v. baar, Mabire bar, Jegu báarân?

\section{road -}

root - Ubi cèrù, Mawa $s \varepsilon d u(\mathrm{R})$, Sokoro sòdú, Dangla tyáàrò, Migama câ:rú, Bidiya cáarà, Mubi kyaaru, Birgit cááró, Toram šaari, Kajakse cara, ?Kujarke gadarra; Kwang kàsár, Kera kósár, Kabalai hàrà, sa:r-hábere, Lele sārā, Sumray sárbō, Ndam sírwé, Tumak $h \bar{\partial} r \bar{a} w<\mathrm{pCh} * \hat{c} o / u H a r-($ Stolbova 1996: 53-54; Ead. 1987: 200: WCh *carw/ya) $=$ *şar- (NEwMAn 1977: 31, \#107; NewMAn \& MA 1966: 238, \#87: *s-rw-) $=*_{2} r w$ "root, vein" $\left(\mathrm{JI}_{1}, 140 \& \mathrm{JI}_{2}, 276-277\right)$.

sand - Ubi yoolè; ?Kabalai kàliňr. $\mathrm{JI}_{1}, 142 \& \mathrm{JI}_{2}, 280-281$ connect the Kabalai form with Lele nàáylā, and further with CCh: Zime Batna nyétl, Masa yétlná; WCh: SBauchi: Geji yelsi id., plus many others.

say - Ubi bògin, Sokoro bókiō.

see - Ubi nayìn, Mawa nê = neyay (R), ?Sokoro géna-gō. Maybe connected with $\mathrm{pCh}$ *Ciyan- or *nah- / *nay- id., both reconstructed on the basis of W \& CCh (Stolbova 1996: 78 and 83 respectively; cf. also WCh *Cayan- \& *nah-; Stolbova 1987: 228 \& 235). NewMAN (1977: $31, \# 111)$ reconstructs pCh *na "to see", also only on the basis of the W \& CCh data (cf. also NEwMAN \& MA 1966: 238, \#90). JI, $144-145$ \& $\mathrm{JI}_{2}, 284-285$ seek a common denominator in $\mathrm{pCh} *$ ngn.

seed - Ubi lobinà, Mawa labən (R), Bidiya luwò. It is questionable, if such forms as CCh: Gisiga hiłfa, Mofu húlfàd; Glavda xúlafà; Margi wüulfüù id. (cf. JI $2,286-287$ \& JI ${ }_{1}$, 146) are related.

sit (down) - Ubi 'unìn, Mawa un (R), Migama nùntù, Mokilko koonè, Mabire untu, Jegu 'un, Birgit 'ùntí; Ndam wúnyə̆< ECh *[u]nt (JSh 230). Newman (1977: 29, \#82) connects Mokilko koonè with W \& CCh forms derivable from $\mathrm{pCh} x^{w}$ on- "to lie down".

skin - Ubi pinyà, Sokoro páńa. Cf. CCh: Hona pana id. (Newman \& MA 1966: 239, \#95).

sleep - Ubi wuyù, Sokoro wóiwói = wó’̀̀ (Sx), Mawa woy- = weyay (R), Mabire oje; Kwang wéy id., Tobanga wōòy "to lie (down)", Kabalai yì "to sleep"; further perhaps Dangla wèdyē, Migama 'òo ̣̂yò, Bidiya 'ooḍy, Birgit 'òọ̣yí, Mubi hèyít = hàyyàdé, Minjile he?ít, Kajakse $a y d \underline{d} u . \mathrm{JI}_{1}, 154 \& \mathrm{JI}_{2}, 298-299$ differentiate $\mathrm{pCh} * w y$ and $* w$ - $d^{y}$.

small - Ubi tedeeki, ?Toram dokoḍit, ?Dormo deiguo.

smoke - Ubi siyò, Mawa si, Sokoro v. sī, Mabire sio, Mokilko sáà, ?Bidiya ziḍà; Kwang v. kisé (L), Kera késé, Gabri gússo, Lele kùsá (WP), Sumray dísa, Tumak hàw. JSh 241 connect with WCh: Ngizim zàwuk, Bade zákúwán id.; CCh: Zeghwana zukè, Gawa zukà, Nakatsa tsuxa id. (Kraft).

\section{stand -}

star - Ubi tímyò, Mawa tìmbi.

stone - Ubi dùrgù.

sun - Ubi pudiyò, Sokoro pío, Mawa pídî, Dangla pàtò, Migama pá:tó, Bidiya páató, Mokilko pèe dó, Mabire pat, Jegu fóot, Birgit fòòtó, Toram fòo, Mubi fà̀t, Minjile fāat, Ka- 
jakse fáti, Kujarke $\Lambda f \wedge r$. Stolbova (1996: 28) reconstructs pCh *fati/a (> WCh *fAtA; see Stolbova 1987: 159) = *fati by Newman (1977: 32, \#126` cf. also Newman \& Ma 1966: 239, $\# 105)=* p-t\left(\mathrm{JI}_{1}, 161 \& \mathrm{JI}_{2}, 312-313\right)$.

swim - Ubi 'òowin, Mawa $\varepsilon w \varepsilon \eta(\mathrm{R})$; perhaps also Mokilko kủubé, kóppé?

tail - Ubi borbilà, Mawa barbal (R), Sokoro búmmel; ?Tumak bámuro; maybe also WCh: Ron: Kulere byéèl id. $\left(\mathrm{JI}_{2}, 316-317\right.$; JSh 260: *bml).

that - Ubi 'ànam, Sokoro ìnu, Mokilko 'êy.

this -

thou (m./f.) - Ubi ci / ke, Sokoro ca, Mawa ki/kó = ci/ko, Dangla kín/kán, Migama kûl kây, Bidiya kinda/kanda, Mokilko kèe/kòn, ?Mabire gimu-ke, Jegu kee/kánêe, Mubi kám/ kín, Minjile kım, Kajakse kımte, ?Kujarke nigi; Kwang gi/kin, ?Kera tam/te, Tobanga jí/mé, Lele digi/dàmè (WP), Sumray à (n)jóm, Tumak ỳ̀/mò.

three - Ubi sùba, Sokoro sùbbá (Sx), Mawa súp, Barain subu, Dangla sûbbà, Migama súbbà, Bidiya subay, Mogum sup, Mabire sup, Jegu sup/b, Birgit súúbù, Toram suuba, Mubi súbà, Minjile subà, Kajakse soop, Kujarke ubo; Kwang sūpáy s ̀̇bày, Kera soope, Kabalai sàp, Lele sùbù, Sumray súbù, Gulei cuba, Miltu sobo, Sarwa sup, Ndam sûp, Tumak suùb (cf. NeWman 1977: 125, apud\#132: ECh innovation $*_{s-} 6-=*_{s-} b$ by $\mathrm{JI}_{1}, 168 \& \mathrm{JI}_{2}, 327$ ).

tongue - Ubi silínyò, Sokoro sólańd-um = sćlèn (Sx), Mawa siliny; Kera kasəl; in other languages without metathesis: Barain lisingo; Dangla léésé, Migama liit = liic-um (J), Bidiya líise, Mokilko 'ilzé, Jegu lèesó, Birgit lì̀sì, Toram liho, Mubi lísí, Minjile 'lîŝ̂, Kajakse liisi, pl. liyàs (A), Kujarke aliyati; Kwang kílisí = keles-úm (L), Nancere kelendem, Gabri kelendin (L), Dormo kélene, Kabalai kólà- = klāndi (L), Lele kīlā-, Sumray dilèsé, Ndam dâs, Tumak dìj. Stolbova (1996: 88) reconstructs pCh *(ha-)lesi-um "(your) tongue" = *ahlasi (NEwmAn 1977: 33, \#134) $=* l s_{3^{-}}\left(\mathrm{JI}_{1}, 169 \& \mathrm{JI}_{2}, 328-329\right)$. Cf. also WCh *ha-lisium (Stolbova 1987: 237).

tooth - Ubi sìnà, Sokoro sónd-im = sən $(\mathrm{Sx})$, Mawa sínó, Barain zengu, Dangla sààyò, Migama sâ:nú, Bidiya séeyo, Mokilko sìntá, pl. siná, Mabire pl. sin, Jegu sàyó, Birgit sàyó, Mubi sìáayj, Kajakse sin, ?Kujarke kiya; Kera kásón, Gabri ksong (L) = kasendi (GD), Dormo gásene, Kabalai kás ònda, Lele kāsīngá, Sumray sándē, Ndam hân = hindam (GD), Tumak hiìn, Sarwa sandam. Stolbova (1996: 57) reconstructs pCh *sina(n) "tooth" (cf. also South Bauchi * sin reconstructed by Stolbova 1987: 261), Newman (1977: 33, \#135): *şan(-). Cf. also Newman \& MA 1966: 240, \#111: ${ }^{*} s-n$ and further $\mathrm{JI}_{1}, 170 \& \mathrm{JI}_{2}, 330-331: *_{s_{3}} n$.

tree - Ubi cùwà, Sokoro tsui, Mawa $s \Lambda w \Lambda=$ səw (R), ?Gabri sehīa (L). JSh 275 connect them with other forms in Chadic, reconstructing *' $t(t)$.

\section{two -}

walk - Ubi ḍ̣, Bidiya ḍàwàn; Kwang d̦ē, Kera ḍć < pCh *taw- (Stolbova 1996: 35-36) $=* d$ ə (Newman 1977: 27, \#59).

warm - Ubi iigiro, ?Mubi kèrit, ?Minjile -kerit, Kajakse kìrí.

water - Ubi 'àmyò, Sokoro mbo $(\mathrm{N})$, Mawa 'ámî, Barain amí, Dangla ámáy, Migama àmmì, Bidiya 'àmay, Mokilko 'à'ú < *'àm-ú, Mabire amba, Jegu 'ám, Birgit 'àmì, Mubi àmé, Minjile aam, Kajakse àmé; Kwang kààm, ká:m, Kera kan, Nancere kā, Gabri kang, kam $(\mathrm{N})=$ kama (GD), Dormo káng, Kabalai ka:mò, Lele kāmāa, Sumray pl. nímī, Ndam nám, Tumak nám, Sarwa nam < pCh *am (Newman 1977: 34, \#142; Newman \& MA 1966: 
240, \#116) = *ymn $\left(\mathrm{JI}_{1}, 176 \& \mathrm{JI}_{2}, 340-341\right)$; cf. also WCh *hama id. and / or North Bauchi *ambi id. (Stolbova 1987: 229 \& 249).

we (ex./in.) - Ubi 'inani, Sokoro ónoy, Mawa neky/é = ne/ea (R), Dangla níin/nìr, Migama nî̀/kéè, Bidiya ninda, Mokilko kàyé/kiné, Jegu nìnée, Mubi ćné, Minjile ènè, Kajakse ante, obj. 'ana (A); Kwang nàn/tàn, Kera áréláy, Tobanga ní/ná .. gay, Lele dàngàngù/dànì, Sumray ándigē, Tumak nà/dì.

what? - Ubi mò, Dangla maa, Mawa mí = me (R), Migama ínimé, Bidiya má, Mokilko 'âmmê, mê, Mabire ja-ma, Jegu mê, Mubi mî̀: Minjile mî, Kajakse maande; Kera ma mó, Nancere $m e(n e)$, Lele $m \bar{e}<* m i / * m ə$ (Newman 1977: 34, \#143; Newman \& Ma 1966: 240, \#117: *m-(n-)).

white - Ubi mòrmìn.

who? - Ubi ('ay) yé, Sokoro ye, besides Mawa weki (R), Dangla waa, Migama wè, Bidiya wá, Mokilko wéè(wé), Jegu wee, ?Kajakse kaye; Nancere wei, Lele wéy (WP) < *wa (Newman 1977: 34, \#146; Newman \& Ma 1966: 240, \#118: *w-(n-)).

woman - Ubi meyè, Mawa mii (R), Sokoro mấa; Nancere tāma, Gabri tama(L), Dormo táma, Kabalai tamă, Lele tāmá, pl. kāmdā (WP), Sumray dèmé, pl. nàmde, Ndam jāmé, cf. dehem (GD), Tumak dèem, Sarwa ndema < pCh *'am- (Stolbova 1996: 31; ead. 1987: 231: WCh *'ama). The closest cognates to the ECh forms seem to occur in WCh: NBauchi: Warji ámái, Tsagu óóméy, Kariya âm, Miya ám, Jimbin ảmá, Mburku dàmà id., although $\mathrm{JI}_{1}, 179$ separate them.

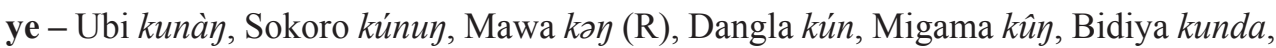
Mokilko kùnè, Jegu kúnéè, Mubi kénć, Minjile kınte, Kajakse 'eetè, obj. kann (A); Kwang kàn, Kera ay, Lele dàngù (WP), Sumray injimge (L), Tumak subj. nì.

\section{CONCLUSION}

Although the incomplete lexicons of some of the languages studied here do not allow their inclusion into the mutual lexicostatistic comparison of all East Chadic languages, the results are convincing enough to determine the position of Ubi in other way than it was indicated in the last edition of Ethnologue, namely together with Sokoro and other close idioms, Mawa and Barain. One argument is the score $60 \%$ of the common basic lexicon for Ubi \& Sokoro, 62\% for Sokoro \& Mawa and even 73\% for Ubi \& Mawa. It is supplemented by the exclusive lexical or word-formation isoglosses connecting Ubi with Sokoro, Mawa and Barain: "bark", "blood", "come", "dry", "foot”, "hand”, "head”, "hear", "knee”, "know", "liver", "long”, "meat”, "night”, "nose”, “red”, “see”, “skin”, “star", "swim”, "tongue", "tree", "who?", "woman", totally 22. Summing up, in the genetic classification of the Chadic languages, Sokoro does not represent a group consisting of only one language, but a cluster represented at least by four idioms: Sokoro, Ubi, Mawa, Barain.

\section{ACKNOWLEDGMENT}

This study originated in cooperation with the Centre for the Interdisciplinary Research of Ancient Languages and Older Stages of Modern Languages (MSM 0021622435) at Masaryk University, Brno, and thanks to the grant No. IAA901640805. 


\section{REFERENCES}

Alıo Khalil. 1989. Lexique Bidiya: une langue centre-africaine (République du Tchad). Frankfurt am Main: Klostermann.

Alıo Khalil. 2004. "Préliminaires à une étude de la langue kajakse d'Am-Dam, de toram du Salamat, d'ubi de Guéra et de masmaje du Batha-Est (Tchad).” In: TAKÁcs 2004: 229-285 [=A].

Bearth Th. et al. (ed.). 1994. Perspektiven afrikanischer Forschung (X. Afrikanistentag). Köln: Köppe, 69-72.

Bender M. Lionel (ed.). 1983. Nilo-Saharan Language Studies. East Lansing: African Studies Center.

BLAŽEK Václav 1994. "Toward Determining the Position of Mokilko within Chadic (a Lexicostatistic Analysis)." In: BEARTH et al. 1994: 69-72.

BlažEK Václav. 2008. "Lexicostatistics Applied to the East Chadic languages.” Folia Orientalia 44, $97-127$.

CAPRILE Jean-Pierre. 1975. Lexique tumak-français (Tchad). Berlin: Reimer.

Doornbos Paul, Bender M. Lionel 1983. “Languages of Wadai-Darfur.” In: Bender 1983: 42-79.

EBert Karen H. 1976. Sprache und tradition der Kera (Tschad). II: Lexikon/Lexique. Berlin: Reimer.

Ebert Karen H. 1987. A First Comparison of Kera and Kwang.” In: Jungraithmayr 1987, 61-70.

Ebobisse Carl 1987. Les verbaux du dangaleat de l'est (Guera, Tchad). Berlin: Reimer [= Ebs].

FÉDRY Jacques 1971. "Masculin, féminin et collectif en dangaléat (groupe "sokoro-mubi" - Tchad)." Africana Marburgensia 10(1), 34-46.

Gaudefroy-Demombynes M. 1907. "Documents sur les langues de l'Oubangui-Chari.” In: Actes du 14e congrès international des orientalistes, 2. Paris, 172-330 [= GD; including Dec $=$ Decorse $]$.

$\mathrm{JI}_{1 / 2}=$ Jungraithmayr Herrmann, IBriszimow Dymitr 1994. Chadic Lexical Roots. I: Tentative reconstruction, grading, distribution and comments; II: Documentation. Berlin: Reimer [J = Jungraithmayr, Sx $=\mathrm{Saxon}]$.

Johnson Eric, Намм Cameron. 2002. Mabire: A Dying Language of Chad. Dallas: SIL International.

JSh = JungraithmaYr Herrmann, Shimizu Kiyoshi. 1981. Chadic Lexical Roots (A first Evaluation of the Marburg Chadic Word Catalogue). Vol. II: Tentative Reconstruction, Grading and Distribution. Berlin: Reimer.

Jungraithmayr Herrmann. 1978. "Gebrochene Plurale im Mubi (Ost-Tschad).” In: Struktur und Wandel afrikanischer Sprachen (Vorträge vom XX. Deutschen Orientalistentag, Erlangen 1977), ed. H. JungRAITHMAYR. Berlin: Reimer, 121-131.

JungraithmaYr Herrmann. 1981. "Über die Mawa (Guera, Tschad) - Ethnographische linguistische Notizen". In: Festschrift zum 60. Geburtstag von P. Anton Vorbichler, Teil 1, ed. I. Hofmann. Wien: Beiträge zur Afrikanistik, 11, 47-70.

JungraithmaYr Herrmann (ed.). 1987. Langues tchadiques et langues non tchadiques en contact en Afrique centrale. Paris: SELAF (Lacito-documents, Afrique 10).

Jungraithmayr Herrmann 1990. Lexique Mokilko. Berlin: Reimer.

Jungraithmayr Herrmann 2004. "Das Birgit, eine osttschadische Sprache - Vokabular und grammatische Notizen.” In: TAKÁCS 2004: 342-371.

JungraithmaYr Herrmann, Adams Abakar. 1992. Lexique Migama. Berlin: Reimer.

Kraft Charles H. 1981. Chadic Wordlists, I-III. Berlin: Reimer.

LuKas Johannes 1937. Zentralsudanischen Studien. Hamburg: Friedrichsen [= L; including AF = A. Friedrich, $\mathrm{N}=$ Nachtigal].

LuKas Johannes 1977. "Das Mukulu.” Afrika und Übersee 60, 1-58, 192-225 [=L].

LWANGA Charles 1985. Vocabulaire dangaleat (parler de l'est). Sarh: Documents du Centre d'etudes linguistiques $[=\mathrm{Lw}]$.

Johnson Eric, Hamm Cameron. 2002. Mabire: A dying Language of Chad. Ms.: SIL International.

Mouchet J. 1950. "Vocabulaires comparatifs de quinze parlers du Nord-Cameroun." Études Camerounaises T. III, No. 29-30, 5-74.

Newman Paul 1977. "Chadic Classification and Reconstructions.” Afroasiatic Linguistics 5(1), 1-42.

Newman Paul, Ma Roxana. 1966. "Comparative Chadic: Phonology and Lexicon.” Journal of African Languages 5(3), 218-251.

Porxomovskij V.Ja. (ed.). 1987. Afrikanskoe istoričeskoe jazykoznanie. Moskva: Nauka.

Roberts Jim. 1994. Lexique Mawa. Ms.: Société Internationale de Linguistique $[=R]$.

SkINNER Neil. 1977. "North Bauci Chadic Languages: Common Roots." Afroasiatic Linguistics 4(1), 1-49.

Stolbova Olga V. 1987. "Sravnitel'no-istoričeskaja fonetika i slovaŕ zapadnočadskix jazykov.” In: PorXOMOvskiJ 1987: 30-268. 
Stolbova Olga V. 1996. Studies in Chadic Comparative Phonology. Moscow: Diaphragma.

TAKÁCs Gábor (ed.). 2004. Egyptian and Semito-Hamitic (Afro-Asiatic) studies in Memoriam W. Vycichl. LeidenBoston: Brill, 229-285 [=A].

Welbegué Christophe, Palayer Pierre. 1982. Lexique lele-français. Sarh: Centre d'etudes linguistiques [= WP].

Allatum die 13 mensis Maii anno 2010 